\title{
Temporal shifting: A hidden key to the skewed peak puzzle
}

\author{
Su-Cheng Pai ${ }^{a}, *$, Ling-Yun Chiao ${ }^{b}$ \\ ${ }^{a}$ Division of Marine Chemistry, Institute of Oceanography, National Taiwan University, Taipei, Taiwan \\ ${ }^{\mathrm{b}}$ Division of Marine Geology and Geophysics, Institute of Oceanography, National Taiwan University, Taipei, Taiwan
}

Received 11 April 2006; received in revised form 28 October 2006; accepted 3 November 2006

Available online 21 November 2006

\begin{abstract}
The recorder-provided peak position for flow-type chemical instruments has been verified mathematically as being comprised of a "spatiallynon-existent" shift, which is generated due to the relativity in accounting for the detection at a fixed point. This shift, denoted as $\Phi$, can be approximated by $\Phi \approx 0.5 \mu_{t}^{2}$, where $\mu_{t}$ is the temporal expanding coefficient of the system given. For flow injection analysis, the shift is correlated to a longitudinal dispersion coefficient $D$ and the flow speed $u$, i.e., $\Phi \approx D / u^{2}$. For linear chromatography, it is correlated to a dynamic partition ratio $k^{\prime \prime}$ and a scaling factor $f$ of the column used, i.e., $\Phi \approx 0.5 k^{\prime \prime} f$. In combination, the temporal shift can be expressed as $\Phi \approx 0.5 k^{\prime \prime} f+D\left(k^{\prime \prime}+1\right)^{2} / u^{2}$. Although the shift may be small in scale, it provides a clue to decipher the basic parameters from a recorded peak. Under a linear isotherm, this parameter can be estimated readily from an experimental peak following a very simple procedure.
\end{abstract}

(C) 2006 Elsevier B.V. All rights reserved.

Keywords: Flow injection analysis; Linear chromatography; Peak shape; Temporal shift

\section{Introduction}

Many analytical instruments give results as peaks on a recorder, which are frequently skewed to carry a tail. The occurrence of this tailing has been usually explained as the result of non-ideal conditions or defects of the instrumentation, but recent studies [1-3] have proposed a new prospect, i.e., the peak skewness may also be generated from an image-transformation distortion due to the observation made at a fixedpoint. Thus, a next question is raised: "Does the recorder also give a false peak position?" The answer may also be a surprising "Yes," though a direct mathematical derivation is yet to be established.

In describing the peak formation in flow injection analysis (FIA) [1], it has been noticed that the recorded peak position $\left(t_{\mathrm{p}}^{*}\right)$ always appears earlier than the theoretical arrival time $\left(t_{\mathrm{p}}=L / u ; L\right.$ is the length of the tubular channel and $u$ the average flow speed). The difference is not insignificant, and a value of $0.5 \mathrm{~s}$ has been reported in that paper. The "shift" is denoted

\footnotetext{
* Corresponding author at: P.O. Box 23-13, Taipei, Taiwan. Tel.: +8862 23627358; fax: +886223632912.

E-mail address: scpai@ntu.edu.tw (S.-C. Pai).
}

here as

$$
\Phi=t_{\mathrm{p}}-t_{\mathrm{p}}^{*}
$$

A similar phenomenon has also been described in the dimensionless Parcel models [2,3]. On a chromatogram, the location of a peak $\left(t_{\mathrm{r}}\right)$ always appears ahead of the corresponding retention time (denoted as $\tau_{\mathrm{rm}} ; \tau_{\mathrm{rm}}=N_{\mathrm{c}} / v_{\mathrm{m}} ; N_{\mathrm{c}}$ is the dimensionless column length and $v_{\mathrm{m}}$ the dimensionless migration speed) by a small but almost consistent difference:

$\Phi=0.5 k^{\prime \prime}$

where $k^{\prime \prime}$ is a dynamic partition ratio between the stationary and mobile phases at a given flow speed.

Although the principles of the above two techniques are likely different [4], they both point out the same fact that the temporal distortion is not a real mechanism but a product of an axial convolution process, resulting in a "twisted" peak image and a "shifted" peak position. If this is correct, then Eqs. (1) and (2) may be linked together in a theoretical way. The scale of the temporal shift is closely related to the expanding nature of the sample zone, for which a faster zone broadening rate will lead to a larger shifting on the temporal axis, and vice versa. However, this somewhat abstract concept might not be well accepted by many scientists unless the shifting term can be rigorously derived. 


\section{Theoretical}

\subsection{Basic implementation}

An ideal micro-bore tubular channel is implemented as follows: the system is running at a constant flow speed $u$; a detector is located at $L=L_{\mathrm{p}}$; and a small sample plug is injected at a position of $L=0$ at time $t=0$. The sample zone expands while traveling in a longitudinal direction. The zone width, which is characterized by the standard deviation $\sigma_{L}(t)$, is proportional to the square root of time (i.e., $\sigma_{L}(t) \propto \sqrt{t}$ ) or distance $\left(\sigma_{L}(t) \propto \sqrt{L}\right.$, where $\left.L=u t\right)$. An ideal evolution of the sample zone through both distance and time is illustrated in Fig. 1, in which the migration route of the mass center is a straight line, with two thresholds $\left( \pm \sigma_{L}(t)\right)$ on both sides. When the center of the sample zone arrives at the detector at $L_{\mathrm{p}}$ (or at time $t_{\mathrm{p}}=L_{\mathrm{p}} / u$ ), the longitudinal peak standard deviation can be expressed as

$\sigma_{L}\left(t_{\mathrm{p}}\right)=\mu_{L} \sqrt{u t_{\mathrm{p}}}$

where $\mu_{L}$ is defined as the expanding coefficient along the $L$ coordinate, in $\mathrm{cm}^{1 / 2}$ units.

\subsection{Peak curves}

A mass distribution curve along the $L$ coordinate at a given time $t$ was derived as early as 1952 by Lipidus and Amundson [5]. Incorporating also the evolution through time, a complete variation of the mass concentration can be expressed as a twodimensional Gaussian function [6]

$C(L, t)=\frac{A_{L}}{\mu_{L} \sqrt{2 \pi u t}} \mathrm{e}^{-(L-u t)^{2} /\left(2 \mu_{L}^{2} u t\right)}$

where $A_{L}$ is the integrated total peak area along the longitudinal coordinate. A snapshot taken at any fixed time, say $t_{\mathrm{p}}$, yields a

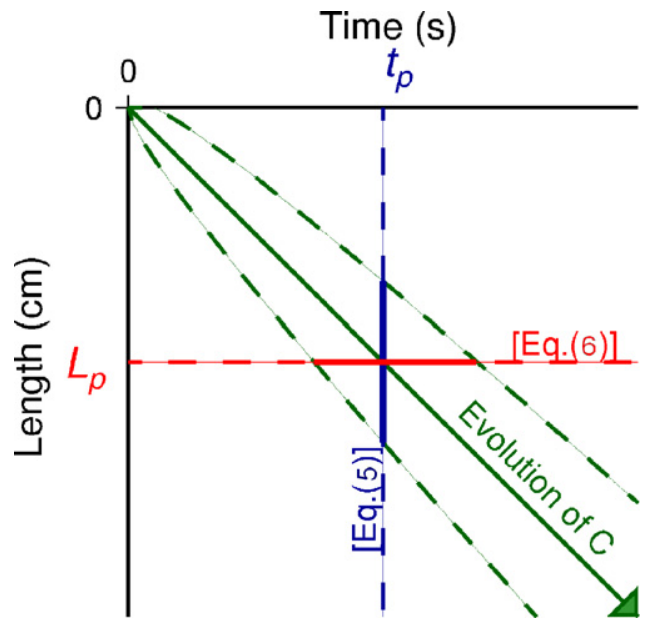

Fig. 1. Evolution of a sample plug on a length vs. time plane. The sample plug is injected at $L=0$ and $t=0$, and a detector is placed at $L=L_{\mathrm{p}}$ that records the arrival of the mass center at $t_{\mathrm{p}}=L / u$. The migration route of the peak summit is marked by a solid line with dashed lines on both sides demarcating the standard deviation $\left( \pm 1 \sigma_{L}\right)$ boundaries. A spatial distribution pattern at $t=t_{\mathrm{p}}$ is represented by Eq. (5) along a vertical line, whereas a temporal curve (Eq. (6)), as might be recorded by the detector at $L=L_{\mathrm{p}}$, is shown along a horizontal line.

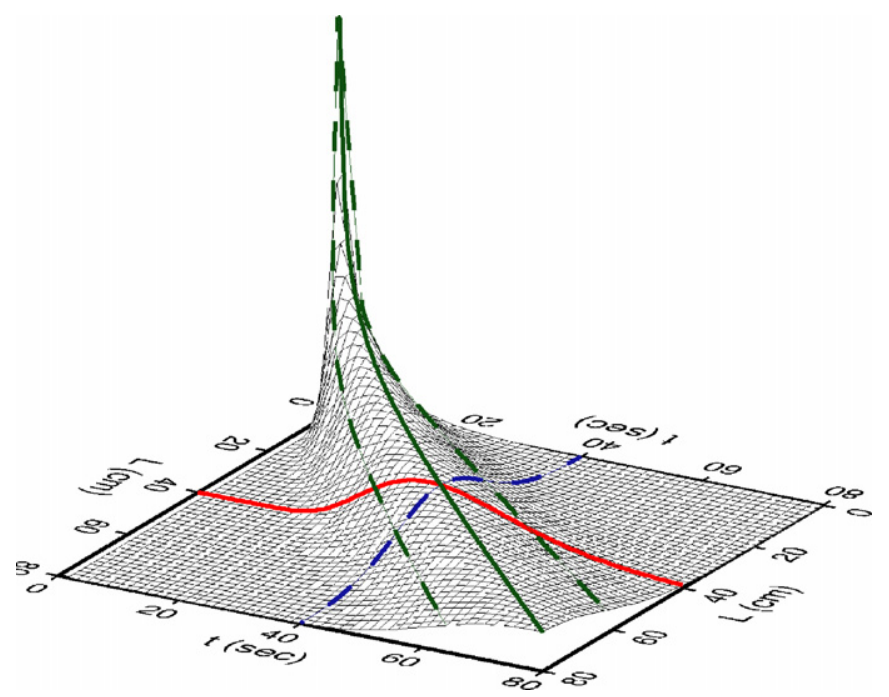

Fig. 2. Three-dimensional perspective of the concentration evolving through both space and time. The diagonal solid line marks the migration route of the mass center, and the dashed lines illustrate the peak broadening. The peak curve at a fixed time $\left(t_{\mathrm{p}}=40 \mathrm{~s}\right)$ marks a snapshot of the spatial pattern, whereas a temporal cross section at a fixed position $\left(L_{\mathrm{p}}=40 \mathrm{~cm}\right)$ depicted by the line mimics the signal recorded on a recorder (see also Fig. 1).

sliced pattern along the $L$ axis as a spatial Gaussian curve (see the example as demonstrated in Fig. 2)

$C(L)=\frac{A_{L}}{\mu_{L} \sqrt{2 \pi u t_{\mathrm{p}}}} \mathrm{e}^{-\left(L-u t_{\mathrm{p}}\right)^{2} /\left(2 \mu_{L}^{2} u t_{\mathrm{p}}\right)}$

On the other hand, what is recorded on the recorder located at $L_{\mathrm{p}}$ is essentially a perpendicular cross section at $\left(L_{\mathrm{p}}, t_{\mathrm{p}}\right)$ (also see Fig. 2). For a temporal view at this point, all spatial peak parameters should be transformed according to the followings: the temporal peak area $A_{t}=A_{L} / u$, the peak position $t_{\mathrm{p}}=L_{\mathrm{p}} / u$, the expanding coefficient on $t$ axis $\mu_{t}^{2}=\mu_{L}^{2} / u$, and the standard deviation $\sigma_{t}(t)=\sigma_{L}(t) / u$. This axial transformation process has been named the "convolution." In another thought, the same function may be derived directly from Eq. (4) by letting the peak position as a constant. Thus, the correct temporal peak curve should be

$C(t)=\frac{A_{\mathrm{t}}}{\mu_{t} \sqrt{2 \pi t}} \mathrm{e}^{-\left(t-t_{\mathrm{p}}\right)^{2} /\left(2 \mu_{t}^{2} t\right)}$

If the spatial curve (Eq. (5)) is normalized to a temporal scale by letting $t=L / u$, then the two curves can be overlapped on one diagram as shown in Fig. 3. In this diagram, the temporal peak position $\left(t_{\mathrm{p}}^{*}\right)$ appears slightly earlier than does the spatial peak, with a slightly higher peak height $h^{*}=C\left(t_{\mathrm{p}}^{*}\right)$ over that of the spatial peak height $h=C\left(t_{\mathrm{p}}\right)$.

\subsection{The temporal shift}

The shifting of the temporal peak summit $t_{\mathrm{p}}^{*}$ from $t_{\mathrm{p}}$ can be derived mathematically by letting the result of the differentiation of Eq. (6) as zero. A $t_{\mathrm{p}}^{*}$ value can be found such that

$\left.\frac{\mathrm{d} C}{\mathrm{~d} t}\right|_{t=t_{\mathrm{p}}^{*}}=0$ 


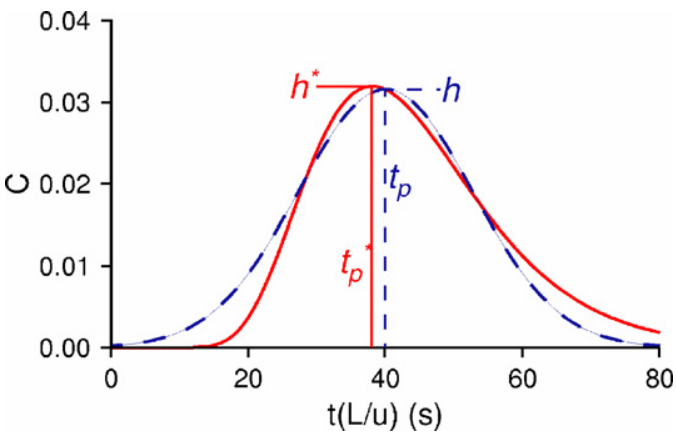

Fig. 3. Overlaying the normalized spatial pattern (Eq. (5) is transformed to a $L / u$ axis, the dashed line) and the temporal peak curve (Eq. (6), the solid line) on the time scale $(t$ or $L / u)$. Assuming $u=1 \mathrm{~cm} \mathrm{~s}^{-1}, A_{t}=1, \mu_{t}=2 \mathrm{~s}^{1 / 2}$ and $t_{p}=40 \mathrm{~s}$, the recorded peak will appear at $t_{\mathrm{p}}^{*}=38.05 \mathrm{~s}$, with an observed peak height of $h^{*}=0.0319$, slightly higher than the expected $h=0.0315$. The temporal shift in this case is $\Phi=t_{\mathrm{p}}-t_{\mathrm{p}}^{*}=1.95 \mathrm{~s}$.

This equation can be easily simplified as

$\left(t_{\mathrm{p}}^{*}-t_{\mathrm{p}}\right)^{2}-2 t_{\mathrm{p}}^{*}\left(t_{\mathrm{p}}^{*}-t_{\mathrm{p}}\right)-\mu_{t}^{2} t_{\mathrm{p}}^{*}=0$

Since the difference between the two peak positions is defined as $\Phi,\left(t_{\mathrm{p}}-t_{\mathrm{p}}^{*}=\Phi\right.$ or $\left.t_{\mathrm{p}}^{*}=t_{\mathrm{p}}-\Phi\right)$, the above equation can be rearranged as

$\Phi^{2}-\left(2 t_{\mathrm{p}}+\mu_{t}^{2}\right) \Phi+\mu_{t}^{2} t_{\mathrm{p}}=0$

The solution for $\Phi$ is therefore

$\Phi=\frac{\left(2 t_{\mathrm{p}}+\mu_{t}^{2}\right)-\sqrt{4 t_{\mathrm{p}}^{2}+\mu_{t}^{4}}}{2}$

If $t_{\mathrm{p}}$ is large enough (or $4 t_{\mathrm{p}}^{2} \gg \mu_{t}^{4}$ ), then the term $\sqrt{4 t_{\mathrm{p}}^{2}+\mu_{t}^{4}} \approx 2 t_{\mathrm{p}}$, the $\Phi$ value will rapidly converge to a fixed threshold (see Fig. 4)

$\Phi \approx \frac{\mu_{t}^{2}}{2}$

Therefore, the observed peak position $t_{\mathrm{p}}^{*}$ should appear theoretically ahead of the actual position $t_{\mathrm{p}}$ and can be estimated by $t_{\mathrm{p}}^{*} \approx t_{\mathrm{p}}-\mu_{t}^{2} / 2$. Reversely, the actual peak position should be at $t_{\mathrm{p}} \approx t_{\mathrm{p}}^{*}+\mu_{t}^{2} / 2$.

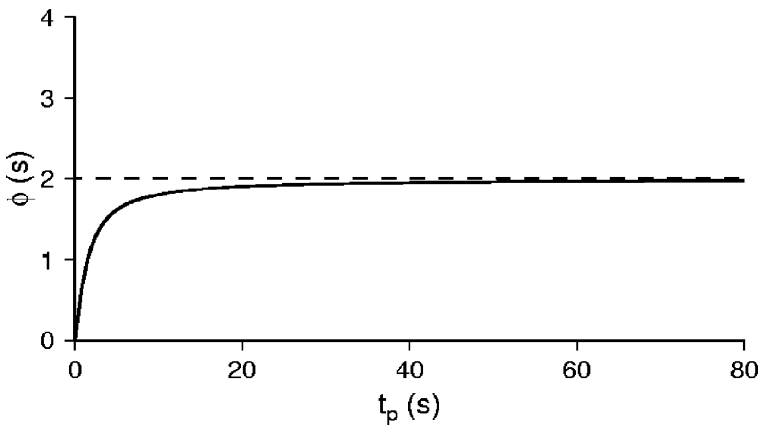

Fig. 4. The temporal shifting $(\Phi)$ as a function of $t_{\mathrm{p}}$ and $\mu_{t}$ as shown in Eq. (10). As $t_{\mathrm{p}}$ grows, it quickly converges to $\mu_{t}^{2} / 2\left(\mu_{t}=2 \mathrm{~s}^{1 / 2}\right.$ in this example).

\subsection{Peak heights}

Also from Fig. 3, the height of a spatial peak at $t_{\mathrm{p}}$ is

$h=\frac{A_{t}}{\mu_{t} \sqrt{2 \pi t_{\mathrm{p}}}}$

But, the temporal peak height (denoted as $h^{*}$ ) should refer to the position at $t=t_{\mathrm{p}}^{*}$

$h^{*}=\frac{A_{t}}{\mu_{t} \sqrt{2 \pi t_{\mathrm{p}}^{*}}} \mathrm{e}^{-\left(t_{\mathrm{p}}^{*}-t_{\mathrm{p}}\right)^{2} /\left(2 \mu_{t}^{2} t_{\mathrm{p}}^{*}\right)}$

The ratio $\left(h^{*} / h\right)$ between the two peak heights is

$\frac{h^{*}}{h}=\sqrt{\frac{t_{\mathrm{p}}}{\left(t_{\mathrm{p}}-\Phi\right)}} \mathrm{e}^{-\Phi^{2} /\left[2 \mu_{t}^{2}\left(t_{\mathrm{p}}-\Phi\right)\right]}$

Numerical verification shows that the first portion $\left(\sqrt{t_{\mathrm{p}} /\left(t_{\mathrm{p}}-\Phi\right)}\right)$ is always larger than 1 , and that the second exponential portion is always smaller than 1 . In combination, $h^{*} / h$ is slightly larger but rapidly merges to 1 when $t_{\mathrm{p}}$ becomes large or $t_{\mathrm{p}} \gg \Phi$ (see Fig. 5). This means that the influence of the temporal distortion effect is comparatively less significant to the peak height than to the peak position.

\subsection{In flow injection analysis}

In a flow injection system, the peak expanding is mainly governed by a longitudinal dispersion-diffusion effect [4]. Although "dispersion" and "diffusion" should occur simultaneously, the former is much larger in scale than the latter when the liquid is flowing. Therefore, the molecular diffusion can be ignored here. The relationship between the peak standard deviation $\left(\sigma_{L}\right)$ at a specific time and the dispersion coefficient $D$ can be expressed as

$\sigma_{L}(t)=\sqrt{2 D t}$

On the temporal scale, the standard deviation becomes

$\sigma_{t}(t)=\frac{\sigma_{L}(t)}{u}=\sqrt{\frac{2 D}{u^{2}} t}$

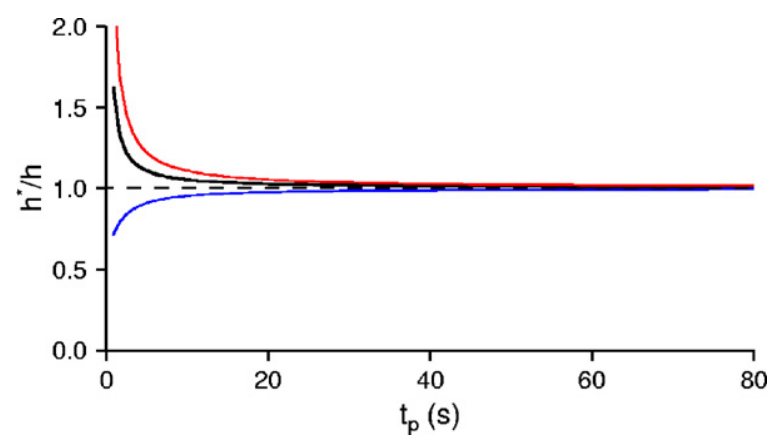

Fig. 5. The ratio of the apparent peak amplitude $\left(h^{*}\right.$ at $\left.t_{\mathrm{p}}^{*}\right)$ vs. the expected spatial peak height ( $h$ at $\left.t_{\mathrm{p}}\right)$ as evaluated according to Eq. (14) $(\Phi=2 \mathrm{~s})$. The first portion $\left(\sqrt{t_{\mathrm{p}} /\left(t_{\mathrm{p}}-\Phi\right)}\right.$, represented by the top line) compensates the variation of the second portion (the bottom line), and renders $h^{*} / h$ to converge quickly to 1 . 
The temporal expanding coefficient $\mu_{t}$ is

$\mu_{t}=\sqrt{\frac{2 D}{u^{2}}}$

If $t_{\mathrm{p}}$ is large enough, the temporal shift (Eq. (11)) can be approximated by

$\Phi \approx \frac{D}{u^{2}}$

Thus, the temporal shift $\Phi$ is linearly proportional to the dispersion coefficient $D$ of the flow system, and also the reciprocal of the square of the flow speed $u$.

\subsection{In linear chromatography without dispersion}

In linear chromatography, the expansion of a sample zone can be considered as a "bulk" retention mechanism, which is correlated to a dynamic equilibrium ratio $k^{\prime \prime}$ of a substance between the stationary and mobile phases [2,3]. The dispersion effect is usually minimal due to the compact packing of the resin bed in the column (i.e., $D$ can be treated as zero here). The flow speed of the mobile influent is still $u$, but the peak migration speed should be $u_{\mathrm{m}}=u /\left(k^{\prime \prime}+1\right)$. Spatially, the mass retention time is defined as $t_{\mathrm{rm}}=L_{\mathrm{c}} / u_{\mathrm{m}}$, whereas temporally, the peak position should be found at $t_{\mathrm{r}}$ on the recorder. The difference is the temporal shift: $\Phi=t_{\mathrm{r}}-t_{\mathrm{rm}}$.

The expanding rate of the zone variance on the longitudinal axis is

$\frac{\mathrm{d} \sigma_{L}(t)^{2}}{\mathrm{~d} t}=\frac{k^{\prime \prime}}{\left(k^{\prime \prime}+1\right)^{2}} u^{2} f$

where $f$ (unit: $\mathrm{s}$ ) is a factor characterizing the physical conditions of the system in use (to be small for high efficiency and vice versa). In fact, $f$ is equivalent to the temporal scaling factor $\Delta t$ in the discrete parcel model $[2,3,7]$ (Note: $u^{2} f$ equals $\Delta L^{2} / \Delta t$ or $u \Delta L$ ). Thus, the "longitudinal" standard deviation of the sample zone when reaching the detector at $t_{\mathrm{rm}}$ is

$\sigma_{L}\left(t_{\mathrm{rm}}\right)=\sqrt{\frac{k^{\prime \prime} u^{2} f}{\left(k^{\prime \prime}+1\right)^{2}} t_{\mathrm{rm}}}$

The "temporal" standard deviation is obtained by further dividing it by the migration speed.

$\sigma_{t}\left(t_{\mathrm{rm}}\right)=\frac{\sigma_{L}\left(t_{\mathrm{rm}}\right)}{u_{\mathrm{m}}}=\sqrt{k^{\prime \prime} f t_{\mathrm{rm}}}$

Thus, the temporal expanding coefficient is

$\mu_{t}=\sqrt{k^{\prime \prime} f}$

When $t_{\mathrm{rm}}$ is large enough, the peak position shift at $t_{\mathrm{rm}}$ can be approximated by

$\Phi=\frac{2 t_{\mathrm{rm}}+k^{\prime \prime} f-\sqrt{4 t_{\mathrm{rm}}^{2}+\left(k^{\prime \prime} f\right)^{2}}}{2} \approx \frac{k^{\prime \prime} f}{2}$

In the dimensionless Parcel model when the physical factor $f$ is unique (both $\Delta L$ and $\Delta t$ are defined to be 1), the shift will be approximately $0.5 k^{\prime \prime}$. This gives a rational explanation to the numerical verification of the temporal shift (Eq. (2)) in that model $[2,3]$.

\subsection{With both retention and diffusion effects}

If both retention and diffusion effects co-exist in a chromatographic system, one may consider to add the individual variances together and to give a summation

$\sigma_{L}^{2}=\sigma_{\text {initial }}^{2}+\sigma_{\text {retention }}^{2}+\sigma_{\text {dispersion }}^{2}$

Assuming that the injection size is minimal, i.e., $\sigma_{\text {initial }}^{2} \approx 0$, the longitudinal variance is a linear function of time. When the peak arrives at the detector at $t_{\mathrm{rm}}=L_{\mathrm{p}} / u_{\mathrm{m}}$, the variance becomes

$\sigma_{L}\left(t_{\mathrm{rm}}\right)^{2}=\frac{k^{\prime \prime} u^{2} f}{\left(k^{\prime \prime}+1\right)^{2}} t_{\mathrm{rm}}+2 D t_{\mathrm{rm}}$

The combined temporal expanding coefficient is therefore

$\mu_{t}=\sqrt{k^{\prime \prime} f+\frac{2 D\left(k^{\prime \prime}+1\right)^{2}}{u^{2}}}$

and the temporal shift can be expressed by

$\Phi \approx \frac{k^{\prime \prime} f}{2}+\frac{D\left(k^{\prime \prime}+1\right)^{2}}{u^{2}}$

The first term on the right-hand side of the equation is a contribution of the retention nature of the column, whereas the second term is attributed mainly to the dispersion effect, with an additional $\left(k^{\prime \prime}+1\right)^{2}$ term acting as a magnifying factor. If $k^{\prime \prime}=0$, it fits FIA cases with no retention; whereas when $D$ approaches zero, the equation matches the ideal case of linear chromatography.

\subsection{Predicting the scale of shifting}

Even though the temporal shift for both FIA and chromatography has been developed in mathematical ways, it can be almost sure that the scale of such a shift is much larger for FIA than chromatography. The reason is clear; the dispersion coefficient $D$ is usually large (e.g. at $10^{1}-10^{2} \mathrm{~cm}^{2} \mathrm{~s}^{-1}$ level) in a hollow tubing but small in a packed column (e.g. at $10^{-2}-10^{-1} \mathrm{~cm}^{2} \mathrm{~s}^{-1}$ level). The difference can be of several orders of magnitude. Besides, both Eqs. (23) and (27) contain a scaling factor $f$, which is proportional to the height equivalent to a theoretical plate (HETP). For an efficient chromatographic column (with large plate number), both the plate height and $f$ are small. If $D$ is also small, then the scale of the temporal shift that is calculated by Eq. (27) would only be as little as in millisecond units. On the other hand, the scale of shifting for FIA is more significant and can be as large as few seconds.

\section{Restoration of an experimental peak}

Following Section 2.4, the crossover of the spatial and temporal peaks at the summits (where $h^{*} \approx h$ ) provides a "magic" key to retrieve the experimental expanding coefficient (denoted 
as $\mu_{\exp }$ to distinguish it from the theoretical $\mu_{t}$ ). Three basic parameters can be obtained by the recorder: the experimental peak area $A_{\text {exp }}$ (equivalent to $A_{t}$ ), the peak position $t_{\text {exp }}$ (equivalent to $t_{\mathrm{p}}^{*}$ ) and the apparent peak height $h_{\exp }$ (equivalent to $\left.h^{*}\right)$. The empirical expanding coefficient can be estimated by $\mu_{\exp }=A_{\exp } / h_{\exp } / \sqrt{2 \pi t_{\exp }}$. Then, by taking $\Phi=\mu_{\exp }^{2} / 2$, a temporal shift is obtained:

$\Phi \approx \frac{A_{\exp }^{2}}{4 \pi t_{\exp } h_{\exp }^{2}}$

With this value, together with $A_{\exp }, t_{\exp }$ and $\mu_{\exp }=\sqrt{2 \Phi}$, a restoration equation is derived from Eq. (6)

$C(t)=\frac{A_{\exp }}{\sqrt{4 \pi \Phi t}} \mathrm{e}^{-\left(t-t_{\exp }-\Phi\right)^{2} /(4 \Phi t)}$

This curve should generate a very similar shape to the experimental peak with almost identical peak height and peak position, as it has been demonstrated in the earlier work [1]. This quick restoration process is very simple, and it does not require knowing the channel or column length, flow speed, diffusion coefficient, void time, partition ratio, or even the scaling factor. Since the retention and diffusion effects are combined here as a single expanding behavior, the same treatment may apply to both FIA and linear chromatographic peaks.

Many previous researchers have already tried to use empirical Gaussian functions to generate skewed peak shapes, but their focus was mainly based on the modification of the "standard deviation" term [8-11] without altering the experimentally obtained peak height and recorded peak position. In this study, the merit is essentially laid on the modification of the "position" term. The consideration of the temporal shift makes the peak function more comprehensible in terms of physical meaning.

\section{Conclusion}

Temporal shift is a hidden but surprising parameter. It happens to be nearly half of the square of the temporal expanding coefficient $\left(\Phi \approx 0.5 \mu_{t}^{2}\right)$ or half of the temporal variance expanding rate $\left(\Phi \approx 0.5 \mathrm{~d} \sigma_{t}^{2}(t) / \mathrm{d} t\right)$ during the migration process. In FIA, it may also be termed as the "temporal diffusivity." This finding opens a new insight into the skewed peak problems, as the zone broadening and temporal shift can be shown to be so closely related in such a simple way.
In analytical chemistry textbooks, the apparent peak position ( $t_{\mathrm{p}}^{*}$ for FIA, $t_{\mathrm{r}}$ for chromatography) obtained from the recorder has been customarily treated as a fundamental measurement of that system. If the observed peak position implies a shift from its real position in the spatial domain, then the traditional way of calculating the dispersion coefficient $D$ (or the $k^{\prime \prime}$ value for chromatography) would carry a small residue. This residue will be accumulated in the further development of other parameters. It would also cause misinterpretations in explaining an experimental peak shape.

The temporal shifting has not been identified until recently, probably due to the intuitive impression of most scientists that the observed peak position $\left(t_{\mathrm{r}}\right)$ should equal "length/speed" $(t=L / u)$, while neglect mass evolves simultaneously through both spatial and temporal dimensions. Mathematical derivation, as provided in this paper, would be a key to further clarifications of existing theories. Application of the present concept on the measurements of several physico-chemical parameters in a real flow injection system will be presented in a separate paper immediately following this one.

\section{Acknowledgments}

The authors would like to thank S.C. Wong, Y.H. Lai and G. Irby for their valuable discussion. Opinions provided by the editorial board and two anonymous reviewers are also appreciated. This work was partially supported by National Science Council, Taipei, Taiwan, under contract no. NSC94-2611-M-002-015.

\section{References}

[1] S.C. Pai, J. Chromatogr. A 950 (2002) 271

[2] S.C. Pai, J. Chromatogr. A 988 (2003) 233

[3] S.C. Pai, J. Chromatogr. A 1028 (2004) 89.

[4] J. Růžička, G.D. Christian, Analyst 115 (1990) 475.

[5] L. Lipidus, N.L. Amundson, J. Phy. Chem. 56 (1952) 984.

[6] S. Golshan-Shirazi, G. Guiochon, in: F. Dondi, G. Guiochon (Eds.), Theoretical Advancement in Chromatography and Related Separation Techniques, Kluwer Academic Publishers, Dordrecht, 1991.

[7] S.C. Pai, C.S. Chern, L.Y. Chiao, J. Chromatogr. A 1018 (2003) 125.

[8] V.B. Di Marco, G.G. Bombi, J. Chromatogr. A 931 (2001) 1.

[9] J. Li, J. Chromatogr. A 952 (2002) 63.

[10] J.R. Torres-Lapasio, J.J. Baeza-Baeza, M.C. Garcia-Alvares-Coque, Anal. Chem. 69 (1997) 3822.

[11] R.D. Caballero, M.C. Gacia-Alvares-Cogue, J.J. Baeza-Baeza, J. Chromatogr. A 954 (2003) 59 